\title{
Editorial
}

\section{Type 2 (Insulin-Independent) Diabetes - Fibres and Flushers}

\author{
A. G. Cudworth \\ Department of Diabetes, St. Bartholomew's Hospital Medical College, London, England
}

Recent developments concerning the aetiology of diabetes have focussed considerable attention on the pathogenetic factors which may be operating in "juvenile-onset" insulin dependent or Type 1 diabetes. In this disease evidence is accumulating that HLA-linked genes may predispose to an immune mediated destruction of the beta cells which is perhaps initiated by viruses or other environmental factors [1]. These advances are exciting, but it is the "maturity onset", "insulin independent" Type 2 diabetic who dominates our clinics. What has happened with respect to this disease which continues to expand with alarming prevalence in our own society, particularly in recently 'Westernised' ethnic groups? The nature of Type 2 diabetes has certainly prompted much research and speculation, but the only safe conclusion that can be drawn is that we are dealing with a multifactorial condition. There are two areas, however, which look potentially interesting: these can be summarized as 'fibres' and 'flushers'.

It is easy to be cynical, but the evidence relating to the possible causes of the relative insulin deficiency and other disturbances which characterize this syndrome is unconvincing. Against this background of ignorance it is perhaps not surprising that since the introduction of the sulphonylureas and biguanides approximately 25 years ago, there has not been a single major therapeutic advance in the management of Type 2 diabetes. One possible exception to this has been a recent reawakening of interest in dietary fibre. In the acute experimental situation, the addition of guar or pectin to a meal has an 'insulin sparing' effect with a concommitant decrease in blood glucose [2]. A more difficult question is whether the decreased quantity of crude fibre in the 'Western' diet coupled with a corresponding increase in highly refined foods, has an aetiological relationship with the development of either or both obesity and Type 2 diabetes, par- ticularly in ethnic groups who have been exposed to rapid environmental change.

Although evidence is lacking which unequivocally links prevalence of this disease with reduced dietary fibre, it remains an interesting hypothesis. Pursuing this idea, it is worth considering the 'thrifty genotype' concept in a functionally different way compared with that initially described by Neel (1962) [3]. Thus, in an environment where food is limited or unaltered by cooking or modern 'processing', natural selection may have operated to favour a metabolic genotype which provides maximum storage of energy-giving substrates. A rapid change in the environment resulting in relative over-nutrition with calorie rich, highly refined foods could lead to the development of obesity. In other words, this metabolic genotype would now be inappropriate. This would have considerable effects in recently primitive peoples who have been unable to adjust genetically in a few generations to the factors that ancestors of Western peoples had much more time to cope with.

Whereas obesity and diabetes are particularly prevalent in groups who are undergoing rapid social change, it is also true that not all obese people develop glucose intolerance or abnormalities of insulin secretion. Therefore it is necessary to postulate that another factor has to be present which influences the capacity of the beta cells to respond to glucose stimulation. Is this component also genetically determined? Almost certainly it is because of the striking familial aggregation of Type 2 diabetes $[4,5,6]$. The association of obesity with Type 2 diabetes is well accepted, but also it has been clearly demonstrated in a carefully conducted study that the prevalence of this disease among siblings was inversely related to the degree of obesity present in the probands [7], emphasising the heterogeneity which exists in Type 2 diabetes. Thus, obesity is just one of the triggering 
factors in subjects who possess the major genetic susceptibility.

If, therefore, the basic defect in this disease is closely related to the inherent control of the beta cells to respond to an unlimited demand, it might appear that we are still entangled in the same old arguments $-i$. e. are the earliest detectable changes compatible with relative hyperinsulinaemia [8], which might be an adaptive response to over-nutrition, or, as argued by others [9], is there a genetically determined poverty of insulin response to a glucose signal? A speculative answer to this dilemma has emanated from the recent fascinating studies of the chlorpropamide-alcohol flush (CAF) phenomenon, and whether this could reflect a possible relationship between the physiological control of beta cell secretion and certain naturally occurring neuro-transmitters. It must be emphasised that there is no evidence to support this idea although undoubtedly investigators will now be stimulated to test this hypothesis.

The facial flushing precipitated by alcohol experienced by a substantial proportion of patients on chlorpropamide is well established [10]. It is present before the development of diabetes and can be produced on first exposure to the drug. The astute observation that this reaction might have a genetic basis has led the King's College Hospital group to a series of interesting findings. Using a simple challenge test they observed that the CAF reaction is an inherited trait which is an important genetic marker in Type 2 diabetes $[11,12]$. Thus, diabetic identical twins, irrespective of whether one or both twins were diabetic, were concordant for the CAF test. More important however was the demonstration that in families with a dominant pattern of inheritance for mild diabetes, the CAF phenomenon was nearly always present in association with the disease, and in two families there was direct transmission through three generations.

In a carefully analysed population study, Leslie and Pyke have further shown that the CAF reaction provides additional evidence for the existence of genetic heterogeneity in diabetes in general as well as within Type 2 diabetes. There was a striking prevalence $(51 \%)$ of facial flushing in a large series of classical Type 2 diabetics compared with a $10 \%$ frequency of the reaction in Type 1 diabetics which was identical to the prevalence in healthy subjects. Of particular interest is the strong association of the CAF with a family history of Type 2 disease. Eightyone per cent with an affected first degree relative produced the reaction compared with $31 \%$ who had no family history of diabetes. In the families with a dominant pattern of diabetes it is independent of the age of onset of the disease [12]. This suggests that
MODY ('maturity-onset' diabetes in a young person) is not a separate entity, but belongs to the syndrome of a mild form of diabetes which has a pronounced familial aggregation and which may develop at all ages. An important question is whether this is really genetically and aetiologically distinct from the diabetes of CAF positive patients without a significant positive family history. This cannot be fully resolved, but one interpretation is that the gene or genes which determine the CAF reaction is closely linked with one of the major susceptibility genes in Type 2 diabetes. The high prevalence of the disease suggests that several factors are likely to be involved. Therefore, the existence in a family of, say, two or three genes which contribute to the overall predisposition might simulate a dominant mode of inheritance, and produce a variable pattern in the age of onset. There are a small number of affected relatives $(13 \%)$ in such families who did not flush, but as pointed out by Pyke and Leslie [12], the challenge test employed was relatively crude and therefore subclinical reactions may have been missed.

More accurate information has been obtained by studying the rise in facial skin temperature which occurs maximally one hour after the alcohol challenge. Twenty-seven CAF positive patients studied by Leslie et al. [13] showed a mean maximum rise of $1.9^{\circ} \mathrm{C}$ compared with a mean rise of $0.3{ }^{\circ} \mathrm{C}$ in 38 CAF negative subjects. However, there appears to be considerable inter-individual variation in this response and it is not clear whether there is a bimodal distribution which could provide evidence for a genetic polymorphism controlling this reaction. Further observations or the measurement of other parameters of the CAF reaction may help to resolve this question.

A new development in the CAF study has emanated from experiments which suggest that the reaction could be due to an increased sensitivity to endogenous opiates [13]. It was noticed that the chlorpropamide alcohol flushing is very similar to that obtained after infusion with an enkephalin analogue with opiate activity. Furthermore, infusion of this analogue produced a rise in facial temperature of at least $1^{\circ} \mathrm{C}$ in nine $\mathrm{CAF}$ positive patients, which was uniformly higher than the more transient rise observed in CAF negative subjects. A particularly important finding was the demonstration in three subjects tested that the CAF response could be abolished by the infusion of the specific opiate antagonist, naloxone [13]. Although these are preliminary results, the blocking activity appears to be independent of age, sex and the presence or absence of glucose intolerance.

The interest in these observations is that 
enkephalin analogues and other substances with opiate activity such as morphine and beta-endorphin, are all known to have an effect on insulin response and glucose handling. Could, therefore, those naturally occurring penta-peptides with opiate-like properties be involved in the pathogenesis of Type 2 diabetes? Although this is highly speculative, and on first sight slightly crazy, there are good reasons for considering this idea. First, insulin receptors are widely distributed throughout the brain and CNS, frequently adjacent to the sites of opiate receptors [14], and it is possible that they may have a functional inter-relationship. Evidence in support of this is that morphine and beta endorphin produce hyperglycaemia via a central effect. In contrast, administration of enkephalin analogue to humans has been shown to produce a slight reduction in blood glucose [15] which suggests that these neurotransmitters may have different effects on peripheral receptors.

It has also been shown that opiate alkaloids and endogenous opioid peptides are capable of influencing islet cell hormonal secretory activity, probably by interaction with specific receptor sites [16]. These workers also showed that naloxone blocked the morphine induced changes in glucagon and somatostatin secretion, and reduced insulin response by approximately $50 \%$. No alterations in basal activity of these hormones could be demonstrated with naloxone. It is tempting to speculate that the influence of opiate peptides or other neurotransmitters on insulin regulation could be one of the mechanisms which explains the well described phenomenon of the "low insulin responders" who are genetically predisposed to the development of Type 2 diabetes [9].

It has also been recognised that patients with mild Type 2 diabetes, particularly those with a very strong family history, are relatively free of diabetic complications. This has led to yet another intriguing observation in relation to the chlorpropamide-alcohol flush reaction [17]. Thus, $54 \%$ patients who were CAF negative had retinopathy compared with $24 \%$ of CAF positive subjects comparable in age and duration of diabetes. Furthermore, severe retinopathy was confined mostly to the CAF negative group.

In conclusion, the CAF phenomenon has produced a new impetus to the understanding of the genetic basis to a major subgroup of Type 2 diabetes, and may give not only a method for identifying those likely to develop diabetes but also lead to new ideas in the approach to treatment.

\section{References}

1. Cudworth, A. G., Bottazzo, G. F., Doniach, D.: Genetic and immunological factors in Type 1 diabetes. In: Immunological aspects of diabetes mellitus. Irvine, W. J. (Ed.). Edinburgh: Teviot Press 1979

2. Goulder, T. J., Alberti, K. G. M. M.: Dietary fibre and diabetes. Diabetologia 15, 285-287 (1978)

3. Neel, J. V.: Diabetes mellitus: a "thrifty" genotype rendered detrimental by "progress". Am. I. Hum. Genet. 14, 353-362 (1962)

4. Tattersall, R. B., Fajans, S. S.: A difference between the inheritance of classical juvenile-onset and maturity-onset type diabetes of young people. Diabetes 24, 44-53 (1975)

5. Irvine, W. J., Toft, A.D., Holton, D. E., Prescott, R. J., Clarke, B. F., Duncan, L. J. P.: Familial studies of Type I and Type II idiopathic diabetes mellitus. Lancet 1977 II, 325-328

6. Cudworth, A. G.: Type 1 diabetes mellitus. Diabetologia 14, 281-291 (1978)

7. Köbberling, J.: Genetic heterogeneities within idiopathic diabetes. In: The genetics of diabetes mellitus. Creutzfeldt, J., Köbberling, J., Neel, J. V. (Ed.), pp. 79-87. Berlin, Heidelberg, New York: Springer 1975

8. Reavan, G.M., Olefsky, J.M. Relationship between heterogeneity of insulin responses and insulin resistance in normal subjects and patients with chemical diabetes. Diabetologia 13, 201-206 (1977)

9. Luft, R, Efendic, S.: On the pathogenesis of maturity-onset diabetes mellitus. Acta Diabetol. Lat. 15, 1-15 (1978)

10. FitzGerald, M. G., Gaddie, R., Malins, J. M., O'Sullivan, D. J.: Alcohol sensitivity in diabetics receiving chlorpropamide. Diabetes 11, 40-43 (1962)

11. Leslie, R. D. G., Pyke, D. A.: Chlorpropamide-alcohol flushing: a dominantly inherited trait associated with diabetes. Br. Med. J. 1978 II, 1519-1521

12. Pyke, D. A., Leslie, R. D. G.: Chlorpropamide-alcohol flushing a definition of its relationship to non-insulin-dependent diabetes. Br. Med. J. 1978 II, 1521-1522

13. Lestie, R. D. G., Pyke, D.A., Stubbs, W. A.: Sensitivity to enkephalin as a cause of non-insulin-dependent diabetes. Lancet 1979 I, 341-343

14. Havrankova, J., Roth, J., Brownstein, M.: Insulin receptors are widely distributed in the central nervous system of the rat. Nature 272, 827-829 (1978)

15. Stubbs, W. A., Delitala, G., Jones, A., Jeffcoate, W.J., Edwards, C. R. W., Ratter, S. S., Besser, G. M., Bloom, S. R., Alberti, K. G. M. M.: Hormonal and metabolic responses to an enkephalin analogue in normal man. Lancet 1978 II 1225-1227

16. Ipp, E., Dobbs, R., Ungar, R. H.: Morphine and $\beta$-endorphin influence the secretion of the endocrine pancreas. Nature $\mathbf{2 7 6}$, 190-191 (1978)

17. Leslie, R. D.G., Barnett, A.H., Pyke, D. A.: Diabetic retinopathy and chlorpropamide alcohol flushing. Lancet 1979 I, 997-999

A. G. Cudworth, M. D., PH. D., F. R. C.P.

Department of Diabetes

St. Bartholomew's Hospital Medical College

West Smithfield

London, E.C. 1

England 